\title{
Shockable Rhythm Detection Based on Sample Entropy
}

\author{
YangXiao-li, ${ }^{1, a^{*}}$, Li Zhenwei ${ }^{1, \mathrm{~b}}$, Hu Zhigang ${ }^{1, \mathrm{c}}$ \\ ${ }^{1}$ Medical Technology and Engineering School \\ Henan University of Science and technology, Luoyang, China \\ aYangxiaoli39@163.com, 'lzwcsu@126.com, `hu.robert@163.com
}

Keywords: Shockable rhythm, sample entropy, complexity

Abstract. Based on the principle that different rhythm corresponds to different levels of system complexity, study on shockable rhythm (ShR) has been conducted, especially for the sample entropy-based ShR detection method which has been compared with the established complexity method by detecting all data in the CUDB and VFDB database, the result of which suggested that this was an effective detection method for Shockable Rhythm.

\section{Introduction}

Shockable rhythm (ShR) consists of Ventricular Tachycardia (VT) and Ventricular Fibrillation (VF) ${ }^{[1-3]}$. Electrical defibrillation at early stage is an effective therapeutic method for VF. Automated External Defibrillator (AED) can increase the survival rate of SCD out hospital. While the key step for AED to achieving its function is by quickly and accurately detecting ShR. In this study, we focus on sample entropy-based ShR detection method, which may have theoretical and practical importance ${ }^{[4,5]}$.

\section{Sample entropy algorithm}

Following Grassberger' study, Richman has developed a statistic, Sample Entropy (SampEn), which is distinct from approximate entropy and does not count self matching ${ }^{[6,7]}$.

The definition of sample entropy is illustrated combining with calculation process. Consider the first $\mathrm{N}-\mathrm{m}$ vectors in the sequence with length of $\mathrm{N}$ and embedding dimensions of $\mathrm{m}$ and $\mathrm{m}+1$, ensuring that when $1 \leq i \leq N-m$, vector $X_{m}(i)$ of $\mathrm{m}$ dimension and $X_{m+1}(i)$ of $\mathrm{m}+1$ dimension are meaningful.

1) If original data is set as:

$u(1), u(2), \mathrm{L} u(N)$

for a total of $\mathrm{N}$ points.

2) A group of $m$ dimensional vectors is comprised by continuous serial number: from $X_{m}(1)$ to $X_{m}(N-m)$, where:

$X_{m}(i)=[u(i), u(i+1), \mathrm{L} \quad u(i+m-1)]$

$i=1,2, \mathrm{~L}, N-m$, These vectors represent $\mathrm{m}$ continuous u values since point $\mathrm{i}$.

3) Define the distance between $X_{m}(i)$ and $X_{m}(j)$ as ${ }^{d}\left[X_{m}(i), X_{m}(j)\right]$, which denotes the maximum among differences of corresponding elements, that is:

$d\left[X_{m}(i), X_{m}(j)\right]=\max (|u(i+k)-u(j+k)|)$

Where $k=0,1, \mathrm{~L}, m-1 ; i, j=1,2, \mathrm{~L}, N-m, j \neq i$ 。

4) If threshold $\mathrm{r}$ was given, for each value of $i \leq N-m$, the number of $d\left[X_{m}(i), X_{m}(j)\right]$ which is less than $r$ (referred as template matching number) is counted and the ratio between this number and $N-m-1$ is calculated, which is noted as: 


$$
B_{r}^{m}(i)=N^{m}(i) /(N-m-1)
$$

The average to all $i$ values is expressed as

$$
B^{m}(r)=(N-m)^{-1} \cdot \sum_{i=1}^{N-m} B_{r}^{m}(i)
$$

5) The dimension is increased to $m+1$, a group of $m+1$ dimension vectors is comprised by continuous serial number: from $X_{m+1}(1)$ to $X_{m+1}(N-m)$, where

$$
X_{m+1}(i)=[u(i), u(i+1), \mathrm{L}, u(i+m)]
$$

These vectors represent $m+1$ continuous $u$ values since point $i$.

6) The distance between $X_{m+1}(i)$ and $X_{m+1}(j)$ is defined as $d\left[X_{m+1}(i), X_{m+1}(j)\right]$, which is the maximum among the differences of corresponding elements, that is:

$$
d\left[X_{m+1}(i), X_{m+1}(j)\right]=\max (|u(i+k)-u(j+k)|)
$$

Where $^{k}=0,1, \mathrm{~L}, m ; i, j=1,2, \mathrm{~L}, N-m, j \neq i$.

7) Threshold $\mathrm{r}$ is given, for each value in the range $r \leq N-m$, the number of $d\left[X_{m+1}(i), X_{m+1}(j)\right]$ which is less than $\mathrm{r}$ is counted, and the ratio between this number and $N-m-1$ is calculated, which is noted as

$$
B_{r}^{m+1}(i)=N^{m+1}(i) /(N-m-1)
$$

The average to all $i$ values is expressed as:

$$
B^{m+1}(r)=(N-m)^{-1} \cdot \sum_{i=1}^{N-m} B_{r}^{m+1}(i)
$$

Theoretically, the sample entropy of this sequence is:

$$
\operatorname{SampEn}(m, r)=\lim \left\{-\ln \left[B^{m+1}(r) / B^{m}(r)\right]\right\}
$$

When $\mathrm{N}$ is a finite value, the result achieved in accordance with the above process is the estimated sample entropy with sequence length of $\mathrm{N}$, which is noted as:

$$
\operatorname{SampEn}(m, r, N)=-\ln \left[B^{m+1}(r) / B^{m}(r)\right]
$$

The physical meaning of sample entropy is similar with the approximate entropy. The lower of the sample entropy results in the higher of the sequence self similarity; the higher of the value of the sample entropy results in the more complexity of the sample sequence. The major difference between sample entropy and approximate entropy is that: in the approximate entropy calculation process, it compares the data with itself, by counting the number of the templates matched with itself to prevent the emergence of $\ln (0)$ during calculation to further avoid deviation; while for sample entropy, it does not count the self match values, which allows faster calculation speed ${ }^{[8]}$. As entropy is a measure for new information generation rate, there is no meaning for the comparison between the data and itself.

\section{The relationship between the value of $\mathrm{m}$ and $\mathbf{r}$ in sample entropy calculation}

In the MIT-BIH Malignant Ventricular Arrhythmia Database (VFDB), for Premature Ventricular Complex (PVC), VT, VF, Ventricular Flutter (VFL), and Asystole (ASYS), two segments of signal each with signal length of $24 \mathrm{~s}$ and 6000 sample points were gathered, for conducting band pass filter at $1-30 \mathrm{~Hz}$ as the frequency of data acquisited by AED is $1-30 \mathrm{~Hz}$, then the value of $\operatorname{SampEn}$ with $r=0.01 \sim 1$ were calculated when m equals to 2 and 10. For $m=2$, the values of SampEn for PVC, VFL, VT, and VF are listed in increasing sequence; while for the SampEn curve of 
ASYS, it was mingled among VFL, VT, and VF. When $r=0.01$ and approximates to 1 , the SampEn curves for VF, and VT are crossed with VFL and PVC; for the rest cases, they all can be separated. Particularly when $r=0.1 \sim 0.2$, the SampEn curves for VF, VT and VFL, PVC are separated clearly.

\section{Process of sample entropy-based ShR detection}

The process of sample entropy-based ShR detection is:

1) Signal segmentation: firstly, the signal is segmented with each 4 seconds (including 1000 sampling points) as a segment.

2) Signal preprocessing: the signal was filtered by $1-30 \mathrm{~Hz}$ butter band pass filter. As the frequency band of output signal by AED are in the range of $1-30 \mathrm{~Hz}$, this filter can acquire the detection effect more close to AED. The characteristic amplitude-frequency curve is shown on the figure.

3) Calculation of sample entropy: the values of SampEn were calculated when $m=2, r=0.15$ and $^{m}=3, r=0.4$.

4) Discrimination: when $m=2, r=0.15$, the threshold was set as $Y 1=0.4$; when $m=3, r=0.4$, the threshold was set as $Y 2=0.14$. Generally, threshold is an empirical value. If one value of sample entropy is greater than threshold, it is judged as ShR, otherwise NShR.

\section{Detection result and conclusion}

For all data in the VFDB database and CUDB database, performance detection has been conducted by two kinds of method with each continuous 4 seconds as a data segment; in the VFDB database, the number of ShR segment is 7252, NShR is 38276; while in the CUDB database, the number of ShR segment is 3305, NShR is 13813. In the CUDB database, there is no data in some sample points, which are eliminated directly. While for some segments, they both contain ShR and NShR, then it is right whether they were judged as ShR or NShR. For these segments, there are 528 in the CUDB database, and 544 in the VFDB database. The threshold of the reference and the detection results by these two methods are shown on table 1 and 2 . Through analysis, we found that sample entropy-based ShR detection method has good effect, the accuracy rate of which is 12 percentages higher than the method based on complexity and complex rate ${ }^{[9,10]}$; therefore, sample entropy-based ShR detection method is effective in ShR detection.

Table 1 References threshold in these two methods

\begin{tabular}{|c|c|c|}
\hline \multicolumn{2}{|c|}{ Method } & $\begin{array}{l}\text { reference } \\
\text { threshold }\end{array}$ \\
\hline \multicolumn{2}{|c|}{$\begin{array}{l}\text { Method based on complexity and } \\
\text { complex rate }\end{array}$} & 0.173 \\
\hline ShR detection & $\mathrm{M}=2, \mathrm{r}=0.15$ & 0.4 \\
\hline sample entropy & $M=3, r=0.4$ & 0.14 \\
\hline
\end{tabular}


Table 2 Accurate rate of these two methods

\begin{tabular}{ccc}
\hline Methods & \multicolumn{2}{c}{ Accurate rate } \\
& CUDB & VFDB \\
\hline $\begin{array}{c}\text { Method based on } \\
\text { complexity and complex } \\
\text { rate }\end{array}$ & 0.58206 & 0.585649 \\
$\begin{array}{c}\text { ShR detection method } \\
\text { based on sample entropy }\end{array}$ & 0.704401 & 0.712233 \\
\hline
\end{tabular}

\section{References}

[1]Hunt SA, Abraham WT, Chin MH, Feldman AM, Francis GS, Ganiats TG, Jessup M, Konstam MA, Mancini DM, Michl K: ACC/AHA 2005 guideline update for the diagnosis and management of chronic heart failure in the adult: a report of the American College of Cardiology/American Heart Association Task Force on Practice Guidelines (Writing Committee to Update the 2001 Guidelines for the Evaluation and Management of Heart Failure): developed in collaboration with the American College of Chest Physicians and the International Society for Heart and Lung Transplantation: endorsed by the Heart Rhythm Society,J.Circulation (2005)112:154.

[2]Hua W, Zhang LF, Wu YF, Liu XQ, Guo DS, Zhou HL, Gou ZP, Zhao LC, Niu HX, Chen KP: Incidence of sudden cardiac death in China: analysis of 4 regional populations,J.Journal of the American College of Cardiology,(2009)54:110.

[3]Brugada J, Della Bella P, Berruezo A, Bogun F, Scheinman MM, Wolpert C: Electrocardiogram Features of Ventricular Tachycardia/Ventricular Fibrillation as Expression of the Underlying Mechanisms and Site of Origin[J]. Ventricular Tachycardia/Fibrillation Ablation: The State of the Art Based on the Venicechart International Consensus Document,(2009)79.

[4]Lindsay BD: Eliminating Triggers of Ventricular Fibrillation: The Past, Present, and Future,J. Journal of the American College of Cardiology,(2009)54:529.

[5]Gerard B: It Started With Einthoven: The History of the ECG and Cardiac Monitoring,J. AACN Advanced Critical Care, (2010)22:93.

[6]Gilden DL, Marusich LR: Contraction of time in attention-deficit hyperactivity disorder[J]. Neuropsychology,(2009) 23:265.

[7]Yan J, Wang Y, Li F, Yan H, Xia C, Guo R,Analysis and classification of wrist pulse using sample entropy. In. IEEE,(2009 )609-612.

[8]Alcaraz R, Rieta J: A novel application of sample entropy to the electrocardiogram of atrial fibrillation,J. Nonlinear Analysis: Real World Applications,11(2010)1026-1035.

[9]Zhang X, Zhu Y, Thakor N, Wang Z: Detecting ventricular tachycardia and fibrillation by complexity measure,J. IEEE Transactions on biomedical engineering,46(1999)548:555.

[10]Zhang Hongxuan, Zhu Yisheng: DETECTION OF VENTRICULAR TACHYCARDIA AND FIBRILLATION BASED ON COMPLEXITY MEASURE AND COMPLEXITY RATE [J]. CHINESE JOURNAL OF BIOMEDICAL ENGINEERING,20(2001)423-429. 